\title{
Tuberculose em presídios brasileiros: uma revisão integrativa da literatura
}

\author{
Tuberculosis in Brazilian prisons: \\ an integrative review of the literature
}

\author{
Mariana Soares Valença ${ }^{1}$ \\ Lia Gonçalves Possuelo ${ }^{2}$ \\ Marta Regina Cezar-Vaz ${ }^{3}$ \\ Pedro Eduardo Almeida da Silva ${ }^{4}$
}

${ }^{1}$ Centro de Ciências da Vida e da Saúde, Universidade Católica de Pelotas (UCPel). R. Gonçalves Chaves 373, Centro. 96015-560 Pelotas RS Brasil.mariana.valenca@ ucpel.edu.br

${ }^{2}$ Departamento de Biologia e Farmácia, Universidade de Santa Cruz do Sul. Santa Cruz do Sul RS Brasil.

${ }^{3}$ Escola de Enfermagem,

Universidade Federal do

Rio Grande (FURG). Rio

Grande RS Brasil.

${ }^{4}$ Faculdade de Medicina,

FURG. Rio Grande RS

Brasil.

\begin{abstract}
The scope of this study was to organize the knowledge produced on tuberculosis in Brazilian prisons in a systematic manner. A comprehensive review was conducted in the Medline and Lilacs databases and the SciELO electronic library using the key words: "Tuberculosis," "Prisons" and "Brazil." Of the 61 records found, 33 were evaluated (28 duplicated records) and 21 included in the review (12 excluded according to the criteria adopted). These studies contribute effectively to improve the knowledge regarding the scale of the disease among inmates, as well as to the choice of screening and diagnostic methods most appropriate to the prison setting. The incidence rates and prevalence of active and latent tuberculosis are presented, in addition to data on the profile of drug and genotype susceptibility of the clinical results. Awareness of the data presented highlights the need to adopt measures aimed at case detection, treatment and follow-up. It is suggested that the new challenges for scientific research should be linked to the development of specific knowledge about dealing with the problem in an environment full of specificities such as a prison.
\end{abstract}

Key words Tuberculosis, Prisons, Diagnosis, Multidrug-resistant tuberculosis, Genotype
Resumo O objetivo deste estudo foi sistematizar o conhecimento produzido sobre tuberculose em presídios brasileiros. Foi realizada uma revisão integrativa nas bases Medline, Lilacs e biblioteca eletrônica SciELO, com os descritores: "Tuberculosis", "Prisons" e "Brazil". Entre os 61 registros encontrados, 33 foram avaliados (28 duplicados) e 21 incluídos na revisão (12 excluídos segundo critério). Verifica-se que os estudos contribuem efetivamente para conhecer a magnitude da doença entre detentos e para a escolha dos métodos de triagem e diagnóstico mais adequados ao cenário prisional. São fornecidos índices de incidência e prevalência de tuberculose ativa e latente, além de dados sobre o perfil de sensibilidade e genotípico dos isolados clínicos. O conhecimento dos dados apresentados reforça a necessidade da adoção de medidas voltadas à detecção, tratamento e acompanhamento de casos. Sugere-se que os novos desafios para a investigação científica, estejam vinculados ao desenvolvimento de saberes específicos acerca do manejo do problema num ambiente repleto de particularidades como o presídio.

Palavras-chave Tuberculose, Prisões, Diagnóstico, Tuberculose Resistente a Múltiplos Medicamentos, Genótipo 


\section{Introdução}

A tuberculose (TB) é uma das principais causas de morbidade e mortalidade relacionadas às doenças infecciosas nos países em desenvolvimento. Um desafio notável para o controle da TB envolve a incidência desproporcional observada entre as populações de maior risco, incluindo a carcerária. No ambiente prisional, essa desigualdade é resultante de fragilidades sociais inerentes ao próprio indivíduo, bem como desse espaço, onde a superlotação, a ventilação deficiente, a nutrição precária, o consumo de drogas e as doenças associadas convivem com precários ou inexistentes serviços de saúde.

Assim como no Brasil, a ocorrência de TB em prisões vem sendo descrita como um alarmante problema de saúde pública em muitos países. A prevalência mundial de $\mathrm{TB}$ entre detentos pode ser até 50 vezes maior do que as médias nacionais ${ }^{2,3}$. Em 2012, a prevalência de TB em todo mundo foi estimada em 169 casos por 100.000 habitantes ${ }^{4}$, enquanto a prevalência média de TB em presídios de diferentes regiões do mundo, entre 1993 e 2011, foi de 1913 casos por 100.000 habitantes 5 .

Mais de 10,2 milhões de pessoas estão reclusas em instituições penais de todo o mundo, com uma taxa de população carcerária de 144/100.000. Os Estados Unidos possuem a maior taxa mundial, 716/100.000 e, o Brasil, com um total de 458.003 presos, possui uma taxa de população prisional de $274 / 100.000^{6}$.

No Brasil, a taxa de incidência de TB estimada para 2012 foi de 46/100.000 habitantes ${ }^{4}$. Entre detentos, esta taxa pode ser mais de vinte vezes superior ao estimado na população livre, correspondendo a $1080,6 / 100.000^{7}$.

Os elevados índices de TB nos presídios e o contingente de pessoas incluídas neste grupo de risco no Brasil justificam o interesse acadêmico por este tema. Esforços têm sido realizados para melhorias no âmbito da saúde prisional e, em relação à TB, o Plano Nacional de Saúde Prisional prevê uma série de ações estratégicas orientadas ao controle da doença ${ }^{8}$. Em 2010, pela primeira vez, o Manual de Recomendações para o Controle da Tuberculose no Brasil incluiu um capítulo específico que contempla recomendações para o diagnóstico, o tratamento e o acompanhamento dos casos de TB nos presídios 9 . Neste contexto de mudanças recentes nas políticas de saúde prisional no Brasil, torna-se essencial a elaboração de estudos que permitam construir sínteses sobre os desenvolvimentos recentes e indiquem as lacunas de conhecimento associadas ao assunto.
O objetivo deste trabalho é sistematizar o conhecimento produzido sobre TB em presídios brasileiros, uma vez que, com respaldo na exploração de registros derivados de revisões, observou-se a inexistência de estudos focalizando o mesmo objetivo. Portanto, foi realizada uma revisão integrativa acerca da temática "tuberculose em presídios brasileiros", partindo da questão norteadora "como o problema da TB em presídios vem sendo abordado em artigos científicos?".

\section{Métodos}

A escolha do método de revisão integrativa sustenta-se pelo fato deste permitir a busca, a avaliação crítica e a síntese das evidências disponíveis sobre o tema investigado, tendo como produto final o estado atual do conhecimento, a implementação de intervenções e a identificação de lacunas que direcionam para o desenvolvimento de futuras pesquisas. A revisão percorreu etapas de elaboração de um protocolo, contemplando a definição da pergunta de revisão, as estratégias para a busca e a seleção de artigos, a avaliação crítica dos estudos, a coleta, a interpretação e a síntese de dados ${ }^{10}$.

Partindo da pergunta norteadora, as seguintes etapas subsequentes foram desenvolvidas:

Estratégias para a busca e seleção de artigos (Figura 1); a pesquisa destes foi realizada no período de janeiro a fevereiro de 2014, nas bases de dados Medical Literature Analysis and Retrieval System Online (Medline) e Literatura Latino-Americana e do Caribe em Ciências da Saúde (Lilacs) e na biblioteca vitual Scientific Electronic Library Online (SciELO). Justifica-se a escolha dessas fontes de dados, pois, enquanto as duas últimas possibilitam direcionar o acesso de periódicos científicos brasileiros, a Medline permite amplo acesso às publicações em periódicos internacionais.

Para o cruzamento dos descritores definidos: "Tuberculosis", "Prisons" e "Brazil", adotou-se a expressão booleana "AND". Não houve restrição quanto à data de publicação e, como critérios de inclusão, consideraram-se artigos originais publicados em inglês, português e espanhol, que abordassem "TB em presídios brasileiros" como tema central, na perspectiva de detentos ou trabalhadores de presídios acometidos por TB ativa ou latente.

A avaliação crítica dos estudos teve como propósito averiguar se a metodologia estava suficientemente descrita e adequada ao alcance dos obje- 


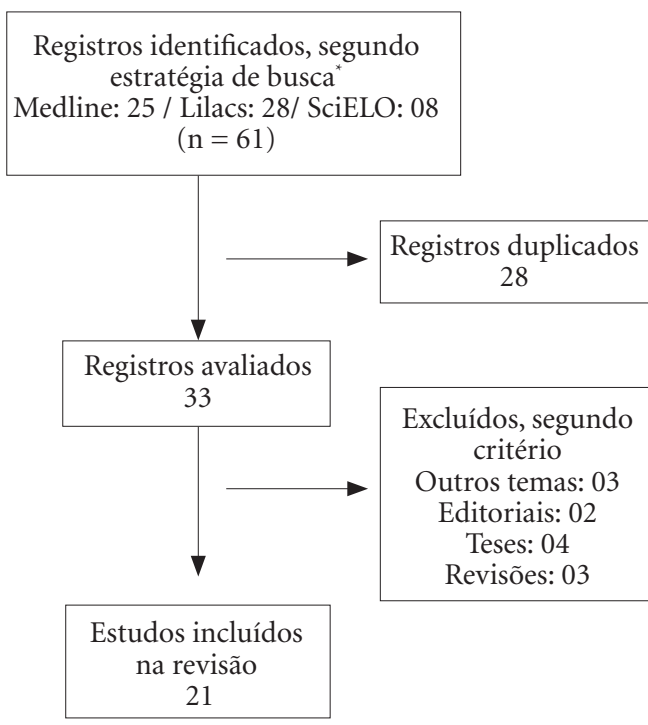

Figura 1. Fluxograma da seleção de estudos que compuseram a revisão integrativa. Medline, Lilacs e SciELO 1996-2013.

"Estratégia de busca Medline realizada via PubMed, adaptada para busca na base Lilacs, realizada via Biblioteca Virtual em Saúde e na biblioteca eletrônica SciELO: (“"tuberculosis"[MeSH Terms] OR "tuberculosis"[All Fields]) AND ("prisons”[MeSH Terms] OR "prisons"[All Fields])) AND ("brazil"[MeSH Terms] OR "brazil"[All Fields]).

tivos propostos, bem como se os resultados estavam compatíveis com a metodologia empregada.

$\mathrm{Na}$ coleta de dados, realizada até março de 2014, foram extraídas informações detalhadas de cada pesquisa, tais como: dados que caracterizam a autoria e o tipo, objetivos, intervenções realizadas no cenário de estudo e evidência dos resultados encontrados.

Os principais resultados dos estudos incluídos foram sistematizados segundo as semelhanças dos dados analisados em cada pesquisa. Originaram-se, a partir disso, as seguintes categorias para a descrição e discussão de resultados: (1) estratégias para a detecção de casos, (2) indicadores de TB ativa e latente em presídios brasileiros, fatores associados, (3) M. tuberculosis isolados de detentos em presídios brasileiros: caracterização do perfil de sensibilidade aos antimicrobianos, (4) dinâmica de transmissão do M. tuberculosis em presídios do Brasil.

A síntese e a interpretação de dados fundamentou-se nos resultados da avaliação crítica dos estudos incluídos. Realizou-se a comparação com o conhecimento teórico, a identificação de conclusões e de implicações resultantes da revisão integrativa ${ }^{10}$. Objetivando minimizar a chance da introdução de vieses, as etapas descritas foram realizadas por dois revisores de forma independente, chegando-se a um consenso nos casos de discordâncias.

\section{Resultados e discussão}

\section{Breve caracterização dos estudos revisados}

Entre os 21 artigos incluídos, 71,4\% (15/21) foram produzidos nos estados do Rio de Janeiro (06/21) ou São Paulo (09/21), no Rio grande do Sul foram elaborados três estudos, enquanto nos estados da Bahia, Espírito Santo e Mato Grosso do Sul, foram publicados um em cada.

Predominantemente, foram desenvolvidos estudos epidemiológicos transversais. Um estudo longitudinal, um qualitativo e um modelo matemático também fazem parte das pesquisas selecionadas para esta revisão integrativa.

A maior parte das pesquisas, $13 / 21(61,9 \%)$, teve como objetivo estimar a incidência e/ou prevalência de TB ativa e/ou latente. Tais dados também puderam ser coletados, mesmo de estudos que não tiveram estes objetivos, possibilitando, assim, observar o panorama geral das taxas de TB encontradas em diferentes presídios brasileiros.

Entre outros objetivos, incluem-se: a descrição de características clínicas e epidemiológicas associadas à $\mathrm{TB}$ e ao desfecho do tratamento, a análise de conhecimento, atitudes e práticas sobre TB, a análise das causas no atraso do diagnóstico de TB em presídios, a caracterização do perfil de sensibilidade aos antimicrobianos e do genótipo dos isolados clínicos, a detecção de fatores de risco associados à TB prisional e a avaliação de diferentes estratégias de busca ativa de sintomáticos respiratórios em presídios (Quadro 1).

\section{Estratégias para a detecção de casos}

Observa-se que os estudos utilizaram diferentes métodos para triagem e diagnóstico de casos suspeitos de TB nos presídios e adotaram, para a produção de dados a serem analisados pela pesquisa, procedimentos recomendados pelo $\mathrm{Mi}$ nistério da Saúde do Brasil ${ }^{9}$. Entre os testes diagnósticos e de triagem, o exame radiológico de tórax ${ }^{11-17}$, a microscopia direta do escarro seguida por cultivo em meio sólido ${ }^{11-23}$ e o teste de sensibilidade aos antimicrobianos (TSA) ${ }^{12,15-19,23}$ foram utilizados por estudos que propuseram alguma 
Quadro 1. Caracterização dos estudos segundo autoria, ano de publicação, objetivos, intervenções e principais achados. Publicações Medline, Lilacs e SciELO 1996-2013.

\begin{tabular}{|c|c|c|c|}
\hline $\begin{array}{l}\text { Autor } \\
\text { Ano }\end{array}$ & Objetivo & $\begin{array}{c}\text { Intervenção } \\
\text { realizada }\end{array}$ & Principais achados \\
\hline $\begin{array}{l}\text { Macedo et } \\
\text { al., } 2013^{24}\end{array}$ & $\begin{array}{l}\text { Descrever características } \\
\text { clínicas e epidemiológicas } \\
\text { associadas com resultado } \\
\text { do tratamento anti-TB. }\end{array}$ & $\begin{array}{l}\text { Sem intervenção } \\
\text { direta. }\end{array}$ & $\begin{array}{l}\text { Abandono do tratamento associado a: presos } \\
\text { jovens, menor escolaridade, uso de álcool, } \\
\text { recidivas e tratamento sem supervisão direta. } \\
\text { TB-MDR }{ }^{+} \text {relacionada a recidiva, abandono, } \\
\text { mudança de centros de tratamento e ter TB } \\
\text { extrapulmonar. }\end{array}$ \\
\hline $\begin{array}{l}\text { Zanini et } \\
\text { al., } 2013^{22}\end{array}$ & $\begin{array}{l}\text { Estimar a prevalência } \\
\text { de TB ativa e identificar } \\
\text { variáveis associadas com } \\
\text { sintomas respiratórios em } \\
\text { uma prisão do Brasl. }\end{array}$ & $\begin{array}{l}\text { Questionário, } \\
\text { microscopia, } \\
\text { cultivo, anti-HIV }\end{array}$ & $\begin{array}{l}\text { Foram considerados sintomáticos respiratórios } \\
68 \% \text { dos detentos avaliados }(178 / 262) \text {, entre } \\
\text { esses, } 25 \text { (14\%) foram diagnosticados com } \\
\text { TB ativa, prevalência de } 9.542 / 100.000 . \\
\text { Baixa escolaridade, uso de álcool e drogas, } \\
\text { reincidência na prisão, TB prévia e HIV } \\
\text { positivo foram associados com a presença de } \\
\text { sintomas respiratórios. A taxa de co-infecção } \\
\text { TB HIV foi de } 60 \% \text {. O desfecho foi óbito em } \\
12 \% \text { dos casos e } 96 \% \text { dos detentos com TB } \\
\text { relataram tratamento irregular. }\end{array}$ \\
\hline $\begin{array}{l}\text { Pereira et } \\
\text { al., } 2013^{23}\end{array}$ & $\begin{array}{l}\text { Estimar a prevalência de } \\
\text { sintomáticos respiratórios } \\
\text { e TB ativa em uma } \\
\text { população privada de } \\
\text { liberdade no Rio Grande } \\
\text { do Sul, Brasil. }\end{array}$ & $\begin{array}{l}\text { Questionário, } \\
\text { escore clínico, } \\
\text { microscopia, } \\
\text { cultivo, TSA`, anti- } \\
\text { HIV. }\end{array}$ & $\begin{array}{l}\text { Um total de } 70 \text { indivíduos }(20,6 \%) \text { foram } \\
\text { considerados sintomáticos respiratórios. Entre } \\
\text { os sintomáticos respiratórios, a prevalência } \\
\text { de tuberculose e HIV foi de } 1,9 \% \text { e } 4,48 \% \text {, } \\
\text { respectivamente. }\end{array}$ \\
\hline $\begin{array}{l}\text { Estevan et } \\
\text { al., } 2013^{11}\end{array}$ & $\begin{array}{l}\text { Estimar prevalência } \\
\text { de TB ativa e ILTB'. } \\
\text { Identificar fatores } \\
\text { associados à ILTB. }\end{array}$ & $\begin{array}{l}\text { Questionário, } \\
\text { PPD }^{\ddagger} \text { exame } \\
\text { radiológico, } \\
\text { microscopia, } \\
\text { cultivo e escore. }\end{array}$ & $\begin{array}{l}\text { Prevalência de ILTB e TB ativa } 49 \% \text { e } 0,4 \% \\
\text { respectivamente. Presença de cicatriz vacinal e } \\
\text { escore da OMS }>5 \text { foram associados à ILTB. }\end{array}$ \\
\hline $\begin{array}{l}\text { Ferreira } \\
\text { Júnior et } \\
\text { al., } 2013^{25}\end{array}$ & $\begin{array}{l}\text { Analisar conhecimento, } \\
\text { atitudes e práticas sobre } \\
\text { TB em uma prisão e nos } \\
\text { serviços de saúde. }\end{array}$ & Questionário. & $\begin{array}{l}\text { Entre detentos, trabalhadores de saúde e } \\
\text { do presídio foram observados conceitos } \\
\text { equivocados sobre a doença. }\end{array}$ \\
\hline $\begin{array}{l}\text { Kuhleis et } \\
\text { al., } 2012^{18}\end{array}$ & $\begin{array}{l}\text { Estimar a incidência, } \\
\text { analisar o perfil } \\
\text { de sensibilidade e } \\
\text { genotipagem dos } \\
\text { isolados. }\end{array}$ & $\begin{array}{l}\text { Questionário, } \\
\text { microscopia, } \\
\text { cultivo, TSA e } \\
\text { genotipagem. }\end{array}$ & $\begin{array}{l}\text { Incidência } 2,9 \% \text {, prevalência, } 3,8 \% .15 \% \text { dos } \\
\text { isolados clínicos resistentes a pelo menos uma } \\
\text { droga, sendo } 87,3 \% \text { pertencentes a algum } \\
\text { cluster. }\end{array}$ \\
\hline $\begin{array}{l}\text { Nogueira } \\
\text { et al., } \\
2012^{19}\end{array}$ & $\begin{array}{l}\text { Estimar a prevalência de } \\
\text { TB ativa ILTB. }\end{array}$ & $\begin{array}{l}\text { Questionário, } \\
\text { PPD, microscopia, } \\
\text { cultivo, TSA. }\end{array}$ & $\begin{array}{l}\text { Prevalência de ILTB e TB ativa } 830,6 / 100.000 \\
\text { e } 1.029,5 / 100.000 \text { respectivamente, } 5 \% \text { dos } \\
\text { casos resistentes na prisão. Já, na delegacia, } \\
\text { prevalência menor de TB ativa 525,7/100.000. }\end{array}$ \\
\hline
\end{tabular}


Quadro 1. continuação

\begin{tabular}{|c|c|c|c|}
\hline $\begin{array}{l}\text { Autor } \\
\text { Ano }\end{array}$ & Objetivo & $\begin{array}{c}\text { Intervenção } \\
\text { realizada }\end{array}$ & Principais achados \\
\hline $\begin{array}{l}\text { Souza et } \\
\text { al., } 2012^{26}\end{array}$ & $\begin{array}{l}\text { Analisar as causas de } \\
\text { atraso no } \\
\text { diagnóstico da TB } \\
\text { na prisão segundo a } \\
\text { experiência do doente } \\
\text { apenado. }\end{array}$ & Entrevistas. & $\begin{array}{l}\text { "O atraso no diagnóstico da TB relaciona-se } \\
\text { à naturalização da desassistência ao sujeito } \\
\text { preso, à interpretação do presídio como um } \\
\text { lugar de morte e sofrimentos e à privação do } \\
\text { direito à saúde para detentos em decorrência } \\
\text { de sua posição nas relações assimétricas de } \\
\text { poder e efeitos ideológicos". }\end{array}$ \\
\hline $\begin{array}{l}\text { Pedro et } \\
\text { al., } 2012^{27}\end{array}$ & $\begin{array}{l}\text { Descrever a detecção do } \\
\text { M. tuberculosis e o perfil } \\
\text { de sensibilidade aos } \\
\text { fármacos. }\end{array}$ & $\begin{array}{l}\text { Sem intervenção } \\
\text { direta. }\end{array}$ & $\begin{array}{l}\text { Total de casos positivos: } 6,9 \% \text {. } \\
4,2 \% \text { de resistência a isoniazida e } 6,2 \% \text { a } \\
\text { rifampicina. Detecção crescenteentre os anos } \\
\text { de } 2003 \text { e } 2006 \text {, de } 2,7 \% \text { para } 6,7 \% \text {. }\end{array}$ \\
\hline $\begin{array}{l}\text { Sanchez et } \\
\text { al., } 2012^{12}\end{array}$ & $\begin{array}{l}\text { Avaliar a circulação do } \\
\text { M. tuberculosis em uma } \\
\text { prisão. }\end{array}$ & $\begin{array}{l}\text { Questionário, } \\
\text { exame radiológico, } \\
\text { microscopia, } \\
\text { cultivo, TSA, } \\
\text { RFLP\|. }\end{array}$ & $\begin{array}{l}84 \% \text { das cepas genotipadas pertenciam a um } \\
\text { dos doze clusters identificados. Dois clusters } \\
\text { principais formados por } 18 \text { e } 21 \text { casos. O } \\
\text { padrão dos genótipos indica infecção exógena } \\
\text { e extensa transmissão do bacilo }\end{array}$ \\
\hline $\begin{array}{l}\text { Nogueira } \\
\text { et al., } \\
2011^{20}\end{array}$ & $\begin{array}{l}\text { Conhecer a prevalência } \\
\text { ILTB entre trabalhadores } \\
\text { comunicantes e não } \\
\text { comunicantes de detentos } \\
\text { com TB. }\end{array}$ & $\begin{array}{l}\text { Questionário, } \\
\text { PPD, microscopia } \\
\text { e cultivo e TSA. }\end{array}$ & $\begin{array}{l}\text { Entre trabalhadores comunicantes e não } \\
\text { comunicantes respectivamente } 62 \% \text { e } 38,9 \% \\
\text { casos de ILTB. Não foram identificados casos } \\
\text { de TB ativa. }\end{array}$ \\
\hline $\begin{array}{l}\text { Vieira et } \\
\text { al., } 2010^{21}\end{array}$ & $\begin{array}{l}\text { Determinar a prevalência } \\
\text { de sintomas respiratórios } \\
\text { e TB através da busca } \\
\text { ativa e estudar fatores } \\
\text { relacionados. }\end{array}$ & $\begin{array}{l}\text { Questionário, } \\
\text { microscopia e } \\
\text { cultivo, coleta } \\
\text { de dados em } \\
\text { prontuário penal. }\end{array}$ & $\begin{array}{l}\text { Prevalência de sintomáticos } 38,7 \%, 39 \text { vezes } \\
\text { superior ao encontrado na população em } \\
\text { geral. Prevalência de TB 1.763/100000, } 35 \text { vezes } \\
\text { maior ao encontrado na população livre. Estar } \\
\text { preso por período maior que } 6 \text { meses foi fator } \\
\text { relacionado a TB. }\end{array}$ \\
\hline $\begin{array}{l}\text { Sanchez et } \\
\text { al., } 2009^{13}\end{array}$ & $\begin{array}{l}\text { Mensurar a prevalência } \\
\text { de TB, identificar fatores } \\
\text { de risco e determinar o } \\
\text { método de triagem mais } \\
\text { adequado entre detentos. }\end{array}$ & $\begin{array}{l}\text { Microscopia e } \\
\text { cultivo entre } \\
\text { aqueles com } \\
\text { alterações } \\
\text { sugestivas de } \\
\text { TB em exame } \\
\text { radiológico. }\end{array}$ & $\begin{array}{l}\text { Prevalência de TB 2,7\%, 32/46 com } \\
\text { confirmação bacteriológica. Associa-se ao } \\
\text { diagnóstico: ser analfabeto, tosse }>3 \text { semanas, } \\
\text { tratamento prévio, viver no subúrbio do RJ. } \\
\text { Baixo rendimento da triagem baseada em } \\
\text { tosse: detecção de } 9 \text { dos } 46 \text { casos. }\end{array}$ \\
\hline $\begin{array}{l}\text { Lemos et } \\
\text { al., } 2009^{14}\end{array}$ & $\begin{array}{l}\text { Determinar a prevalência } \\
\text { de TB ativa e latente em } \\
\text { um hospital penal. }\end{array}$ & $\begin{array}{l}\text { Questionário, } \\
\text { radiologia PPD, } \\
\text { microscopia } \\
\text { e cultivo. }\end{array}$ & $\begin{array}{l}\text { A prevalência de ILTB e TB ativa } 61,5 \% \text { e } \\
2,5 \% \text { respectivamente. Presença de tosse como } \\
\text { determinante de TB ativa. }\end{array}$ \\
\hline $\begin{array}{l}\text { Moreira- } \\
\text { Oliveira et } \\
\text { al., } 2008^{28}\end{array}$ & $\begin{array}{l}\text { Investigar a transmissão } \\
\text { do bacilo entre } 39 \text { presos } \\
\text { com TB em } 4 \text { instituições } \\
\text { penais. }\end{array}$ & $\begin{array}{l}\text { Sem intervenção } \\
\text { direta. }\end{array}$ & $\begin{array}{l}\text { Agrupamento dos isolados em seis perfis } \\
\text { principais. Sugere-se a transmissão do bacilo } \\
\text { tanto entre os presos da mesma, quanto de } \\
\text { diferentes instituições penais. A variável } \\
\text { "tratamento anterior" foi associada com a } \\
\text { transmissão. }\end{array}$ \\
\hline
\end{tabular}




\begin{tabular}{|c|c|c|c|}
\hline \multicolumn{4}{|c|}{ Quadro 1. continuação } \\
\hline $\begin{array}{l}\text { Autor } \\
\text { Ano }\end{array}$ & Objetivo & $\begin{array}{l}\text { Intervenção } \\
\text { realizada }\end{array}$ & Principais achados \\
\hline $\begin{array}{l}\text { Legrand et } \\
\text { al., } 2008^{29}\end{array}$ & $\begin{array}{l}\text { Simular a dinâmica da } \\
\text { TB e avaliar o impacto } \\
\text { de diferentes estratégias, } \\
\text { sobre a prevalência de TB } \\
\text { através de um modelo } \\
\text { matemático. }\end{array}$ & $\begin{array}{l}\text { Sem intervenção } \\
\text { direta. }\end{array}$ & $\begin{array}{l}\text { Estratégia DOTS } \\
2,5 \% \text { da prevalência média de TB. Não houve } \\
\text { efeito significativo associando-se a triagem na } \\
\text { admissão e DOTS. Mas, adicionando-se a estas } \\
\text { duas estratégias, o rastreamento anual por } \\
\text { exame radiológico, seria possível reduzir em } \\
\text { abaixo de } 1 \% \text { a prevalência de TB. }\end{array}$ \\
\hline $\begin{array}{l}\text { Sanchez et } \\
\text { al., } 2007^{15}\end{array}$ & $\begin{array}{l}\text { Comparar prevalência } \\
\text { de } \mathrm{TB} \text {, as características } \\
\text { dos casos e características } \\
\text { sociais e epidemiológicas } \\
\text { dos internos de diferentes } \\
\text { unidades prisionais. }\end{array}$ & $\begin{array}{l}\text { Uso de dados } \\
\text { produzidos em } \\
\text { pesquisa anterior, } \\
\text { publicada em } \\
2005 \text {. }\end{array}$ & $\begin{array}{l}\text { Heterogeneidade da população encarcerada } \\
\text { em três diferentes unidades penais. Taxa de } \\
\text { prevalência na prisão A foi de } 4,6 \% \text {, prisão B } \\
6,3 \% \text { e prisão C } 8,6 \% \text {. Em comparação com } \\
\text { prisão A, os detentos das prisões B e C são } \\
\text { oriundos de comunidades mais desfavorecidas } \\
\text { e têm mais frequentemente, história de } \\
\text { encarceramento anterior e TB prévia. }\end{array}$ \\
\hline $\begin{array}{l}\text { Fournet et } \\
\text { al., } 2006^{30}\end{array}$ & $\begin{array}{l}\text { Desenvolver e avaliar } \\
\text { o desempenho de } \\
\text { escores clínicos para a } \\
\text { identificação de suspeitos } \\
\text { de TB. }\end{array}$ & $\begin{array}{l}\text { Uso de dados } \\
\text { produzidos em } \\
\text { pesquisa anterior, } \\
\text { publicada em } \\
\text { 2005. Avaliação } \\
\text { de três escores } \\
\text { clínicos. }\end{array}$ & $\begin{array}{l}\text { Em relação ao exame radiológico seguido } \\
\text { de microscopia e cultivo: escore da } \\
\text { OMS, sensibilidade 56\%, VPNH 97,2\%, } \\
\text { especificidade } 74,7 \% \text {, VPP } 9,9 \% \text {. Escore OMS } \\
\text { modificado, sensibilidade } 72 \% \text {, VPN 97,8\%, } \\
\text { especificidade } 60 \% \text {, VPP 8,2\%. Novo escore, } \\
\text { sensibilidade } 74 \% \text {, VPN 98,2\%, especificidade } \\
67 \% \text {, VPP 9,6\%. }\end{array}$ \\
\hline $\begin{array}{l}\text { Sanchez et } \\
\text { al., } 2005^{16}\end{array}$ & $\begin{array}{l}\text { Determinar a prevalência } \\
\text { de TB ativa e avaliar o } \\
\text { desempenho de diferentes } \\
\text { estratégias de triagem } \\
\text { para TB. }\end{array}$ & $\begin{array}{l}\text { Radiologia, } \\
\text { microscopia, } \\
\text { cultivo e TSA. } \\
\text { Avaliação de } \\
\text { três métodos de } \\
\text { triagem. }\end{array}$ & $\begin{array}{l}\text { Prevalência de TB 4,6, sendo 2,7\% casos com } \\
\text { confirmação microbiológica. Limitação dos } \\
\text { métodos de triagem baseados em sintomas. } \\
\text { Maior rendimento de rastreamento por exame } \\
\text { radiológico. }\end{array}$ \\
\hline $\begin{array}{l}\text { Oliveira e } \\
\text { Cardoso, } \\
2004^{31}\end{array}$ & $\begin{array}{l}\text { Descrever a incidência } \\
\text { de TB entre detentos } \\
\text { do sistema prisional do } \\
\text { município de Campinas } \\
\text { entre } 1993 \text { e } 2000 \text {. }\end{array}$ & $\begin{array}{l}\text { Sem intervenção } \\
\text { direta. }\end{array}$ & $\begin{array}{l}\text { Maior incidência observada foi de } \\
1.397,62 / 100.000 \text {. Predomínio de TB entre } \\
\text { faixa etária de } 25 \text { a } 34 \text { anos }(62,6 \%) \text { e a AIDS } \\
\text { como doença associada }(49,9 \%) \text {. Microscopia } \\
\text { positiva em } 70,3 \% \text {. O abandono de tratamento } \\
\text { chegou a } 49 \% \text {. Houve cura em apenas } 20,8 \% \\
\text { dos casos no período estudado. }\end{array}$ \\
\hline $\begin{array}{l}\text { Ferreira et } \\
\text { al., } 1996^{17}\end{array}$ & $\begin{array}{l}\text { Caracterizar a incidência } \\
\text { e fatores de risco para } \\
\text { ILTB e TB ativa em uma } \\
\text { prisão feminina com } \\
\text { elevada prevalência de } \\
\text { HIV. }\end{array}$ & $\begin{array}{l}\text { Questionário, } \\
\text { exame radiológico, } \\
\text { PPD, microscopia, } \\
\text { cultivo, TSA, RFLP } \\
\text { e anti-HIV. }\end{array}$ & $\begin{array}{l}\text { 20/378 casos de TB confirmados ( } 5,7 \%), 60,8 \% \\
\text { casos com PPD positivo. } 25 \% \text { das mulheres } \\
\text { infectadas pelo HIV. Conversão do PPD em } \\
29 \% \text { das mulheres HIV positivo e } 32 \% \text { em HIV } \\
\text { negativo. HIV e tempo de encarceramento } \\
\text { maior que } 12 \text { meses foram fatores associados } \\
\text { a TB. }\end{array}$ \\
\hline
\end{tabular}

' ILTB: Infecção Latente por M. tuberculosis; ${ }^{+}$TB-MDR: tuberculose resistente múltiplos fármacos; ${ }^{\dagger}$ PPD Purified Protein Derivative; ${ }^{\circledR}$ TS: teste de sensibilidade aos fármacos; " RFLP: Restriction Fragment Length Polymorphism; ${ }^{* *}$ DOTS: Directly

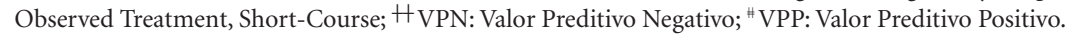


intervenção direta nas unidades penais estudadas (Quadro 1) para coleta de dados primários. Receberão maior destaque nesta seção os estudos destinados à avaliação de diferentes estratégias de detecção de casos em presídios ${ }^{13,15,16,29,30}$, combinando métodos diagnósticos e/ou de triagem para detecção de TB. Outros estudos selecionados segundo critérios de inclusão da presente revisão $\mathrm{O}^{18,22,23,25,26}$ e demais pesquisas citadas a seguir, contribuem para a discussão das estratégias para diagnóstico de TB em presídios.

Em 2005, o exame radiológico de tórax foi realizado em massa para a triagem de detentos com sinais sugestivos de TB, e aqueles que apresentaram anormalidades no exame de imagem tiveram microscopia e cultivo de escarro realizados. Tomando esta intervenção como referência, a prevalência de TB encontrada foi 4,6 \% (42/1052), sendo destes $2,7 \%$ casos com confirmação microbiológica. A partir disso, outras três estratégias para triagem de TB foram simuladas para comparação: (1) tosse por período maior que três semanas; (2) escore clínico proposto pela Organização Mundial da Saúde (OMS) com pontuação maior ou igual a cinco $^{32}$; e, (3) presença de pelo menos um sintoma sugestivo de TB. A estratégia de triagem por tosse com duração $\geq$ três semanas não detectaria $86 \%$ dos casos descobertos com o uso da microscopia isoladamente, enquanto que o exame de cultivo incrementaria em cinco casos diagnosticados, mas, ainda assim, não detectaria 74,4\% dos demais. A segunda e a terceira estratégias igualmente teriam baixo rendimento, 79,1\% dos casos não seriam diagnosticados. Assim, a principal evidência encontrada é que, a triagem de casos suspeitos de TB possibilita uma maior detecção quando realizada através de exame radiológico e não por detecção de sintomas clínicos ${ }^{16}$.

Em 2006, foram avaliadas três estratégias baseadas em escores clínicos, para a identificação de detentos com suspeita de TB. O escore da OMS ${ }^{32}$, um novo escore e o escore da OMS modificado (sendo os dois últimos elaborados/modificados pelos autores) foram estudados em comparação ao exame radiológico. Todos apresentaram baixa sensibilidade em relação ao método de triagem tomado como referência (radiologia), 56\%, $74 \%$ e $72 \%$ respectivamente. A probabilidade de encontrar casos de TB foi baixa (valor preditivo positivo: 10\%). Apesar dos escores apresentarem valor preditivo negativo elevado $(>97 \%)$, tiveram baixa especificidade $(75 \%, 60 \%, 67 \%)^{30}$.

Em 2009, novamente constatou-se o baixo rendimento da triagem baseada em tosse seguida por exame direto e cultivo de escarro: detecção de nove dos 46 casos diagnosticados por exame de imagem associado à avaliação microbiológica (microscopia e cultura) de amostras respiratórias ${ }^{13}$.

Tendo em vista a limitação dos métodos de triagem baseados em sintomas, se justifica o uso de rastreamento inicial por exame radiológico e subsequente realização de microscopia e cultivo de escarro para aqueles com alterações na radiologia de tórax ${ }^{13}$. O rastreio sistemático por radiologia deve ser priorizado em prisões de elevada prevalência ${ }^{15}$ e considerado, ao menos, durante as fases iniciais em que se reforça o controle da $\mathrm{TB}$, a fim de reduzir sua impressionante carga ${ }^{30}$.

Um modelo matemático permitiu simular a dinâmica da TB e avaliar o impacto de três diferentes estratégias, sobre a prevalência: a estratégia Directly Observed Treatment, Short-Course (DOTS), triagem de suspeitos de TB no momento da admissão no presídio e o rastreamento anual por radiologia. O DOTS, mesmo alcançando os objetivos de detectar 70\% dos casos estimados com bacteriologia positiva e curar $85 \%$ daqueles diagnosticados, reduziria, em cinco anos, apenas 2,8\% da prevalência média de TB. Não sendo observado efeito significativo ao incorporar-se a triagem de presos no momento da admissão à estratégia DOTS. Contudo, adicionando-se ao DOTS e à triagem no momento da admissão, o rastreamento anual de TB ativa por exame radiológico de tórax, ou seja, com a combinação de três estratégias de controle da TB, seria possível reduzir sua prevalência para abaixo de $1 \%{ }^{29}$.

Uma vez que se entende a busca ativa para TB como a implementação de ações visando identificar pessoas sintomáticas e realizar diagnóstico de TB ativa, e a busca passiva como a detecção de TB ativa entre pessoas que se apresentam ao serviço de saúde para avaliação dos sintomas ${ }^{33}$, em muitos dos estudos avaliados, as ações de busca ativa e/ou passiva são componentes da etapa de coleta de dados.

A triagem para detecção de TB tem como objetivo primário, aumentar a detecção precoce de casos e, em consequência disso, melhorar os desfechos individuais e diminuir a transmissão do M. tuberculosis na comunidade ${ }^{34}$. Em presídio do sul do Brasil, a busca ativa por meio de sintomas clínicos, o uso do cultivo permitiu o diagnóstico precoce de $25 \%$ dos casos esperados em um ambiente hostil, onde o acesso à saúde é restrito e o sistema de saúde nem sempre é alcançado ${ }^{18}$. Também em outros dois presídios do Sul, 48\% $\%^{22}$ e $66,7 \%{ }^{23}$ dos casos foram diagnosticados apenas por cultura, demonstrando a maior sensibilidade do método em relação a microscopia e permitin- 
do o início do tratamento precoce, antes que o paciente tivesse microscopia positiva.

Verifica-se que existem aspectos que podem dificultar a implementação das medidas recomendadas pela investigação científica. Pois, de acordo com estudo que avaliou o conhecimento, as atitudes e as práticas sobre TB, tanto entre os detentos quanto entre os trabalhadores de saúde e do presídio, foram observados conceitos equivocados sobre a doença ${ }^{25}$.

Além disso, segundo a experiência do doente apenado, o atraso no diagnóstico da TB (fator que contribui para os alarmantes índices da doença em presos), "está relacionado à naturalização da desassistência ao sujeito preso, à interpretação do presídio como um lugar de morte e sofrimentos e à privação do direito à saúde para detentos em decorrência de sua posição" ${ }^{\prime 26}$. Com isso, evidencia-se, ainda, maior descaso de uma doença que já é mundialmente reconhecida como negligenciada, na medida em que a privação de liberdade impõe iniquidade de acesso à assistência em saúde para este grupo populacional. Desta forma, o cenário da pesquisa aponta para outro grande desafio ao controle da TB em presídio: a mudança de concepções sobre o direito à saúde dos apenados. Enxergar o acesso ao sistema de saúde como direito dos detentos e não como concessão do sistema prisional, pode ser o primeiro passo para promover a autonomia dos presos sobre a própria saúde.

\section{Indicadores de TB ativa e latente nos presídios brasileiros, fatores associados}

As taxas de prevalência de TB ativa descritas variaram entre $0,4 \%$ e $8,6 \%$, sendo a menor taxa descrita por estudo realizado no Mato Grosso do $\mathrm{Sul}^{11}$ e, maior, descrita por inquérito epidemiológico desenvolvido no Rio de Janeiro ${ }^{15}$.

Em estudo prospectivo, desenvolvido entre agosto de 2007 e agosto de 2008 no Rio Grande do Sul, a incidência de novos casos chegou a 2,9\% (55/1.900), o que corresponde a uma incidência de 3.789/100.000 detentos. Índice este muito superior ao ser comparado com a capital do estado, Porto Alegre, que por sua vez, também possui uma incidência elevada em relação à do Rio Grande do Sul ${ }^{18}$. Cabe aqui ressaltar que Porto Alegre, entre as demais capitais dos estados brasileiros, é a quarta com maior taxa de incidência de $\mathrm{TB}^{7}$. Dados de prevalência, fornecidos por outros dois estudos em penitenciárias gaúchas são igualmente alarmantes, variando entre $1.898^{23} \mathrm{e}$ $9.542^{22}$ casos de TB por 100.000 detentos.
Utilizando dados laboratoriais retrospectivos, pesquisadores de São José do Rio Preto/SP tiveram como objetivo principal descrever a detecção de bacilo álcool ácido resistente/M. tuberculosis entre amostras pulmonares provenientes de detentos, analisadas por microscopia e/ou cultura. Um percentual de $6,9 \%$ de positividade foi encontrado ${ }^{27}$. Na capital do estado de São Paulo, em 1996, 5,7\% dos casos investigados foram confirmados através do uso de exame radiológico de tórax, microscopia e cultivo de escarro ${ }^{17}$.

Em estudo transversal, utilizando dados laboratoriais referentes a um período de sete anos, a maior incidência observada em um presídio de Campinas/SP chegou a $1397,62 / 100.000^{31}$, enquanto que em Carapicuíba/SP a prevalência de TB entre detentos foi de 1763/100.000 no ano de 2006, sendo 35 vezes superior ao encontrado na população livre ${ }^{21}$.

Encontram-se como fatores associados à TB ativa: a origem de comunidades socioeconomicamente desfavorecidas, possuir menos de um ano de estudo nas séries iniciais ou ser analfabeto, TB prévia $^{13,15,16,22}$, antecedentes de encarceramento $^{13,15-17,22}$, uso de álcool ${ }^{22}$, HIV $^{17,22}$ e AIDS $^{31}$.

A presença de tosse e expectoração também é apontada como fator associado à $\mathrm{TB}$ ativa ${ }^{14} \mathrm{e}, \mathrm{em}$ outro estudo, todos os sintomas respiratórios e sistêmicos, potencialmente relacionados com TB, foram efetivamente associados com casos confirmados. Entretanto, dados clínicos que levam a suspeita da doença foram relatados por detentos com e sem TB, em particular tosse por período superior ou igual a três semanas (definição operacional de sintomático respiratório). Este sintoma foi relatado por $34,9 \%$ e $16,9 \%$ dos presos com e sem TB, respectivamente. Associação estatisticamente significante foi encontrada entre a presença de tosse e tabagismo, relatada em 79\% dos presos sem $\mathrm{TB}^{16}$.

Quatro pesquisas investigaram infecção latente pelo M. tuberculosis (ILTB) através da avaliação do teste tuberculínico (PPD RT23-2TU/0.1 mL). As taxas de prevalência de ILTB apresentadas foram $49 \%{ }^{11}, 60,8 \%{ }^{17}, 61,5 \%{ }^{14}$ e $73 \%{ }^{19}$ em estudos conduzidos entre detentos dos estados de Mato Grosso do Sul, São Paulo e Bahia.

Os autores discutem que as taxas de ILTB entre os presos são superiores à estimada para a população das Américas $(25 \%)^{35}$, superiores também ao encontrado nos Estados Unidos $(14,2 \%)^{36,37}$, país com maior população carcerária do mundo ${ }^{6}$.

O ambiente da prisão é enfatizado nas discussões acerca dos elevados índices de ILTB $^{11,14,17,19}$. 
Por si só, um ambiente de confinamento com alta prevalência da doença em sua forma ativa, a superlotação populacional, a pobre ventilação e iluminação, justifica-se a ocorrência de tantos casos de ILTB diagnosticados, haja vista a transmissão aérea do bacilo.

Além dos fatores ambientais, aspectos comportamentais, onipresentes na população carcerária, contribuem para um maior risco de infecção pelo bacilo. A maioria dos presos tem um histórico de má nutrição e de uso de álcool, tabaco e outras drogas. Uma vez na prisão, estas pessoas continuam a adotar comportamentos de risco, como uso de drogas e sexo sem proteção (ambos os fatores contribuem para a infecção pelo HIV $)^{11}$. Tais aspectos, reconhecidamente encontram-se associados com ILTB ${ }^{4}$.

Alguns autores observaram também uma associação entre a ILTB e os sintomas constantes no escore clínico da OMS. Além do aumento da pontuação no escore, a presença de cicatriz vacinal BCG também foi associada à ILTB. Tal associação pode tanto ocorrer devido à reação cruzada entre a vacina e o PPD, quanto pela infecção por M. tuberculosis ${ }^{11}$.

Em relação a inquéritos tuberculínicos realizados com trabalhadores comunicantes e não comunicantes de apenados com TB ativa, os resultados indicam maior suscetibilidade à ILTB entre trabalhadores comunicantes. São encontradas nesse grupo, taxas de ILTB semelhantes ao relatado em presos $^{20}$. A exposição ocupacional ao bacilo, vivida por profissionais da área da saúde, pode ser comparada ao vivenciado na rotina de trabalho de agentes penitenciários, caracterizando-os também como grupo de risco à infecção pelo M. tuberculosis. Contudo, a reatividade ao PPD, como indicador de ILTB pode ser mais frequente entre trabalhadores de penitenciárias do que entre profissionais que atuam em hospitais, com $62,4 \%{ }^{20}$ e $47,3 \%{ }^{38}$, respectivamente.

\section{M. tuberculosis isolados de detentos de presídios brasileiros: caracterização do perfil de sensibilidade aos antimicrobianos}

O perfil de sensibilidade dos isolados clínicos foi realizado em 31,57\% (6/19) dos presídios estudados, o método das proporções foi utilizado por todas as pesquisas que apresentam resultados de TSA ${ }^{12,15-19,23}$.

No Rio de Janeiro, a avaliação da sensibilidade do M. tuberculosis aos fármacos antituberculose (antiTB) foi realizada em 51,6\% dos pacientes e identificou resistência em 11,1\% (9/81) dos casos; e, entre estes, 2,5\% (2/81) foram identificados como multidroga-resistente (MDR - resistência a pelo menos rifampicina e isoniazida). A resistência primária às drogas, ou seja, pacientes sem história de tratamento prévio para TB, infectados por bacilos resistentes, foi constatada em $4,9 \%$ (4/81) dos casos ${ }^{15}$. No Brasil, o II Inquérito Nacional de Resistência mostrou um aumento da resistência primária à isoniazida (de 4,4 para $6,0 \%$ ) e à isoniazida associada à rifampicina (de $1,1$ para $1,4 \%)^{39,40}$. Esse dado merece especial atenção, porque a transmissão de linhagens resistentes pode ter repercussões negativas na epidemiologia e no controle da TB, uma vez que, há uma menor chance de cura desses pacientes e a terapia é estendida para 18-24 meses, exigindo o uso de fármacos que causam maior toxicidade, necessitando, em alguns casos, ser realizada em unidades de saúde especializadas ${ }^{41}$.

Também em prisões do Rio de Janeiro, 2,2\% (2/89) dos casos foram caracterizados como MDR e 5,6\% (5/89) como monorresistentes (três resistentes à estreptomicina e dois à isoniazida $)^{12}$, tendo sido relatado em outra pesquisa um caso de resistência à isoniazida, entre os 25 detecta$\operatorname{dos}^{16}$. A maior taxa de resistência relatada foi em um presídio do Rio Grande do Sul, 15\% (9/60) dos casos foram resistentes a pelo menos um fármaco, dos quais, 89\% (8/9) apenas à isoniazida ou em combinação com outra droga ${ }^{18}$.

Em 2012, em uma penitenciária de São Paulo, o percentual de casos de resistência foi inferior ao encontrado nas penitenciárias dos estados do Rio de Janeiro e do Rio Grande do Sul, entre vinte isolados, um caso de resistência à rifampicina foi diagnosticado, correspondendo a $5 \%{ }^{19}$.

Não foram encontrados casos de TB causada por cepas resistentes aos fármacos antiTB em duas das pesquisas que realizaram TSA, uma delas em $\mathrm{SP}^{17}$ e outra no Rio Grande do Sul ${ }^{23}$.

São fatores relacionados ao surgimento de casos de TB com cepas resistentes em unidades penais: recidiva, abandono do tratamento e mudança de centros de tratamento pela descontinuidade da tomada de medicação. No Rio Grande do Sul, 96\% dos detentos relataram a interrupção da terapia medicamentosa, dado alarmante devido à maior probabilidade de seleção de cepas resistentes, e pela não interrupção da cadeia de transmissão do bacilo ${ }^{22}$. Em outro presídio de São Paulo (2004), o abandono do tratamento chegou a $49 \%$, havendo cura em apenas $20,8 \%$ dos casos no período estudado ${ }^{31}$. A ocorrência de abandono do tratamento teve maior ocorrência entre os presos mais jovens, com menor 
nível educacional, com história de uso de álcool, recidivas e que não receberam tratamento diretamente observado ${ }^{24}$.

Considerando as pesquisas incluídas em nosso estudo, e apesar do número reduzido de artigos que disponibilizam taxas de resistência do M. tuberculosis na população carcerária, a taxa média de TB resistente em prisões foi 9.7\%. Esta aproxima-se ao observado em inquérito epidemiológico realizado em sete estados do Brasil, em que observou-se $15.3 \%$ e $7,5 \%$ de monorresistência e MDR, respectivamente ${ }^{42}$.

A OMS estima que a incidência global de TB -MDR seja de 3,6\%, entretanto, pouco menos de 30.000 casos de TB-MDR foram notificados em 2009 , isso corresponde a $12 \%$ do total estimado $(250.000)^{43}$. Desta forma, é provável que a grande maioria dos casos não tenha sido diagnosticada ou que tenha havido subnotificação. Pois, o cultivo e o TSA não são realizados rotineiramente na maioria dos países em desenvolvimento, colaborando com a subnotificação de TB-MDR, dificultando a identificação do perfil de resistência, aumentando a morbidade e a mortalidade e propiciando a propagação de cepas resistentes.

Os métodos convencionais de TSA, como o das proporções, utilizado em todas as pesquisas que investigaram o perfil de sensibilidade dos isolados clínicos da presente revisão, apresenta limitações que vão desde uma realização complexa até a demora para a obtenção de resultados. Tal método necessita de isolamento primário do microrganismo, podendo demandar até 60 dias de observação para posterior avaliação do perfil de sensibilidade da cepa. Isso determina que, em alguns casos, o resultado possa ser obtido tardiamente, o que representa risco potencial para os pacientes, trabalhadores das instituições penais, bem como, para a comunidade em geral.

Apesar de estarem disponíveis, os testes rápidos comerciais têm elevado custo e, em geral, requerem maior infraestrutura laboratorial, não sendo possível implementá-los em locais com poucos recursos. Buscando o desenvolvimento de métodos eficazes, baratos e rápidos, têm sido estudados testes de cultivo e TSA não comerciais. Assim, recentemente a OMS reconheceu que os métodos não comerciais como Microscopic Observation of Drug Susceptibility (MODS), Colorimetric Redox Indicator (CRI) e Nitrate Reductase Assay (NRA) possuem evidências científicas suficientes para o seu uso na rotina ${ }^{44}$. A realização de ensaios pragmáticos desses métodos, no contexto dos presídios, seria oportuna para a avaliação e a implementação de alternativas diagnósticas mais rápidas.

\section{Dinâmica de transmissão do M. tuberculosis em presídios do Brasil}

A biologia molecular tem sido usada não apenas para a qualificação do diagnóstico da TB como também como ferramenta para, através da genotipagem dos isolados, realizar estudos de epidemiologia molecular. No Rio de Janeiro, 94 cepas provenientes de detentos com TB foram genotipadas, e utilizando-se Restriction Fragment Length Polymorphism (RFLP) verificou-se que $84 \%$ dos casos eram pertencentes a um dos doze clusters identificados. Os principais clusters possuíam entre 18 e 21 cepas, representando 48,1\% dos casos. O estudo relata, ainda, que todos os casos de resistência pertenciam a um mesmo cluster, com exceção de um com duas cepas sucessivamente isoladas, cada uma pertencente a um cluster, sendo a primeira monorresistente e a segunda MDR. Uma grande proporção de casos agrupados $(68 \%)$ foi diagnosticada pela busca passiva e segundo rastreio radiológico em massa (no primeiro rastreamento não demonstravam evidência de TB). Portanto, este achado sugere que as transmissões intrainstitucionais recentes do bacilo e não as reativações de ILTB contribuem substancialmente para a elevada endemia da doença ${ }^{12}$.

$\mathrm{Na}$ análise de diferenças entre variáveis socioeconômicas, penais e clínicas, destaca-se a história de TB pregressa que, mesmo sem significância estatística, foi duas vezes mais frequente entre os casos pertencentes a clusters. Na maior parte, não houve relação entre o cluster que a cepa foi identificada e o pavilhão de detenção do doente ${ }^{12}$. A dificuldade em determinar essa relação, supostamente está atrelada à grande mobilidade dos presos no interior da instituição. Trocas de celas, galerias ou pavilhões são comumente realizadas por diferentes motivos, entre eles a manutenção da segurança e da disciplina.

A realização de genotipagem por Spoligotyping em uma unidade prisional do Rio Grande do Sul demonstrou que a maior parte dos isolados clínicos $(87,3 \%)$ apresentava genótipos idênticos, sendo, portanto, identificados como pertencentes a algum cluster. Constatou-se também que, entre esta população carcerária, há transmissão de cepas com padrão de genótipos distintos daqueles observados em infecções que acometeram a população livre no mesmo período de estudo ${ }^{18}$.

Os elevados índices de cepas agrupadas encontradas nos presídios do Rio de Janeiro e Rio Grande do Sul refletem a conhecida situação de 
risco de infecção em que vivem presos no sistema prisional, além de ser um claro indicativo de transmissão intramuros do bacilo. A circulação de genótipos distintos daqueles encontrados na comunidade em geral no sul do Brasil deve ser monitorada sob o ponto de vista epidemiológico e microbiológico.

Em um presídio feminino do estado de São Paulo constatou-se que as detentas tinham maior propensão a progredir para TB ativa nos 12 primeiros meses de reclusão. Diante disso, os autores avaliaram se havia rápida progressão para $\mathrm{TB}$ ativa após as detentas serem infectadas dentro da penitenciária ou se já estavam infectadas no momento da reclusão. Onze cepas foram genotipadas por RFLP, sete apresentaram padrão único enquanto quatro distribuíram-se em dois clusters $^{17}$. Não é possível determinar se a maior parte dos casos de TB ativa era decorrente de reativação ou se de infecção recente, uma vez que se observa simultaneamente a ocorrência de diversidade genética das cepas circulantes, o relato de cepas clones com devida relação epidemiológica estabelecida e, também, pela avaliação do teste tuberculínico, que sugere a ocorrência de reativação em 50\% dos casos de TB ativa. Sendo assim, tanto a reativação quanto a infecção recente são situações associadas à ocorrência da doença ativa neste presídio.

Também no estado de São Paulo, um estudo de epidemiologia molecular que envolveu 39 isolados de $M$. tuberculosis, provenientes de quatro diferentes instituições penais, sugere a ocorrência de transmissão do bacilo tanto entre os presos da mesma, quanto de diferentes instituições penais ${ }^{28}$.

Estudos de epidemiologia molecular podem ser usados como ferramentas para monitorar o desempenho dos programas de controle da TB, bem como para produzir informações que complementem as ações de vigilância epidemiológi$\mathrm{ca}^{45,46}$. Esses estudos apontam, além do desempenho do programa, também para questões estruturais e organizacionais deste.

\section{Considerações finais}

O desenvolvimento de estudos científicos é gerador de informações úteis para a implementação de medidas voltadas ao controle da TB, adequadas à realidade prisional, principalmente no que diz respeito ao aperfeiçoamento de diferentes estratégias de detecção de TB e escolha dos métodos mais adequados ao cenário prisional. Destaca-se o maior rendimento da radiologia para a triagem de casos e a contribuição do cultivo para o aumento da detecção de casos.

Em um cenário onde o confinamento determina a rápida propagação do bacilo, sugere-se a realização de ensaios pragmáticos de novos métodos de TSA, visando, assim, a implementação de alternativas para diagnóstico precoce de TB causada por cepa resistente.

A epidemiologia molecular, utilizada ainda por poucos estudos, reflete a consequência das elevadas taxas de TB em presídios, bem como a realidade das condições de encarceramento e adoecimento. Mostra-se, além disso, uma importante ferramenta para o monitoramento do desempenho das medidas de controle e aponta para questões operacionais que podem qualificar o manejo da TB em presídios.

Verifica-se lacunas de conhecimento acerca do real impacto em longo prazo da busca ativa sobre a epidemiologia da TB prisional, embora a possibilidade de um impacto positivo seja apontada por modelos matemáticos. Merece especial atenção, ainda, investigar o quanto a detecção precoce influi na melhoria dos desfechos de TB e reduz a transmissão do bacilo.

Nas pesquisas incluídas nesta revisão, a descrição de como efetivamente se desenvolvem as ações de busca ativa e passiva de TB em um ambiente repleto de particularidades, como as unidades penais, é pobre se comparada com as informações fornecidas sobre outros elementos do processo metodológico, como a descrição de procedimentos laboratoriais.

Embora a condição de reclusão represente uma oportunidade singular para intervir, especificidades do próprio confinamento podem estar relacionadas com fragilidades para a implementação de políticas públicas de saúde. Assim, quais são as potencialidades e as fragilidades para o estabelecimento de ações que visam a minimizar a carga da doença neste cenário? Como se estabelece o fluxo diagnóstico, principalmente no que diz respeito à mobilização dos detentos dentro do presídio para os atendimentos junto à equipe de saúde? Em presídios que não dispõem de infraestrutura laboratorial própria (provavelmente a maioria), como ocorre o encaminhamento das amostras clínicas para o laboratório de referência? Os exames radiológicos são realizados? A comunicação de resultados laboratoriais (tanto entre laboratório-equipe de saúde, quanto entre equipe de saúde-presos) é efetiva para a tomada de decisão clínica? De que forma se dá o seguimento clínico dos casos de TB detectados, considerando as possibilidades de mudança de 
instituições penais, liberdade de presos em tratamento, a realização periódica de exames de controle e o fornecimento de medicações? Neste sentido, estudos científicos podem contribuir para melhor conhecimento da magnitude da TB prisional e eventualmente responder aos principais questionamentos apresentados.

Tem sido sugerido que a TB prisional está associada a características individuais e a condição de vida antes do encarceramento, bem como a fatores ambientais relacionados à reclusão. Considera-se que entre tais fatores ambientais se incluem, além do espaço físico, a questão organizacional do sistema, questões de ordem política e de recursos humanos, tais como as dificuldades para o desenvolvimento de intervenções para detecção precoce dos casos índices, a articulação do sistema prisional com o sistema de saúde e a atuação de equipes multiprofissionais qualificadas para o manejo desse problema no interior dos presídios. Dessa forma, a questão socioambiental é outro aspecto que pode ser objeto de estudo a ser explorado pelas pesquisas com enfoque na $\mathrm{TB}$ em presídios.

Por fim, esta revisão, utilizando os estudos publicados nas bases Medline, Lilacs e biblioteca SciELO possui um objetivo amplo que viabilizou sumarizar uma diversidade de intervenções e desfechos mensurados nos estudos incluídos. A discussão de resultados produzidos por estudos brasileiros, cotejada pela literatura internacional, permite aos profissionais e gestores da área da saúde a obtenção de informações relevantes sobre como o problema da TB em presídio tem sido abordado em diferentes contextos. Estudos com o mesmo objetivo do presente trabalho não foram até então publicados e os resultados aqui descritos podem subsidiar a formulação e a adoção de estratégias voltadas à melhor resolubilidade dos problemas associados à $\mathrm{TB}$ em presídios.

\section{Colaboradores}

MS Valença trabalhou na concepção, no delineamento, na análise e interpretação dos dados e na redação do artigo; LG Possuelo participou da a análise e interpretação dos dados, da revisão crítica e da aprovação da versão a ser publicada; MR Cezar-Vaz trabalhou na versão final a ser publicada e PEA Silva na concepção e delineamento, na sua revisão crítica e na aprovação da versão a ser publicada. 


\section{Referências}

1. Dara M, Grzemska M, Kimerling ME, Reyes H, Zagorskiy A; Coalition for Technical Assistance; International Committee of the Red Cross. Guidelines for Control of Tuberculosis in Prisons (US). Washington: TB/ CTA, ICRC; 2009.

2. World Health Organization (WHO). Status Paper on Prisons and Tuberculosis. Copenhagen: WHO; 2007.

3. Baussano I, Williams BG, Nunn P, Beggiato M, Fedeli $\mathrm{U}$, Scano F. Tuberculosis incidence in prisons: a systematic review. PLoS Med 2010; 7:e1000381.

4. World Health Organization (WHO). Global Tuberculosis Report 2013. Geneva: WHO; 2013.

5. Vinkeles Melchers NVS, van Elsland SL, Lange JMA, Borgdorff MW, van den Hombergh J. State of Affairs of Tuberculosis in Prison Facilities: A Systematic Review of Screening Practices and Recommendations for Best TB Control. PLoS ONE 2013; 8(1):e53644.

6. Walmsley R; International Centre for Prison Studies. World Prison Population List. $10^{\text {th }}$ ed. London: ICPS;2013.

7. Brasil. Ministério da Saúde (MS). Secretaria de Vigilância em Saúde, Programa Nacional de Controle da Tuberculose. Situação da Tuberculose no Brasil. Brasília: MS; 2014.

8. Brasil. Ministério da Saúde (MS), Ministério da Justiça (MJ). Portaria interministerial no 1777, de 9 de setembro de 2003. Diário Oficial da União 2003; 10 set.

9. Brasil. Ministério da Saúde (MS). Secretaria de Vigilância em Saúde. Programa Nacional de Controle da Tuberculose. Manual de recomendações para o controle da tuberculose no Brasil. Brasília: MS; 2010.

10. Mendes KDS, Silveira RCCP, Galvão CM. Revisão integrativa: método de pesquisa para a incorporação de evidências na saúde e na enfermagem. Texto Contexto Enferm 2008; 17(4):758-764.

11. Estevan AO, Oliveira SMVL, Croda J. Active and latent tuberculosis in prisoners in the Central-West Region of Brazil. Rev. Soc. Bras. Med. Trop. 2013; 46(4):515-518.

12. Sánchez AR, Huber FD, Massari V, Barreto A, Camacho LAB, Cesconi V, Saad MH, Larouzé B. Extensive Mycobacterium tuberculosis circulation in a highly endemic prison and the need for urgent environmental interventions. Epidemiol. Infect. 2012; 140(10):1853-1856.

13. Sánchez AR, Larouzé B, Espinola AB, Pires J, Capone D, Gerhardt G, Cesconi V, Procopio MJ, Hijjar M, Massari V. Screening for tuberculosis on admission to highly endemic prisons? The case of Rio de Janeiro State prisons. Int J Tuberc Lung Dis 2009; 13(10):1247-1252.

14. Lemos ACM, Matos ED, Bittencourt CN. Prevalence of active and latent TB among inmates in a prison hospital in Bahia, Brazil. J Bras Pneumol 2009; 35(1):63-68.

15. Sánchez AR, Massari V, Gerhardt G, Barreto AW, Cesconi V, Pires J. Tuberculosis in Rio de Janeiro prisons, Brazil: an urgent public health problem. Cad Saude Publica 2007; 23(3):545-552.

16. Sánchez AR, Gerhardt G, Natal S, Capone D, Espinola $\mathrm{AB}$, Costa W, Pires J, Barreto A, Biondi E, Larouzé B. Prevalence of pulmonary tuberculosis and comparative evaluation of screening strategies in a Brazilian prison. Int J Tuberc Lung Dis 2005; 9(6):633-639.
17. Ferreira MMC, Ferrazoli L, Palaci M, Salles PS, Medeiros LA, Novoa, Kiefer CR, Schechtmann M, Kritski AL, Johnson WD, Riley LW, Ferreira Júnior OC. Tuberculosis and HIV Infection Among Female Inmates in São Paulo, Brazil: A Prospective Cohort Study. J Acquir Immune Defic Syndr 1996; 13(2):177-183.

18. Kuhleis D, Ribeiro AW, Costa ERD, Cafrune PI, Schmid KB, Costa LL, Ribeiro MO, Zaha A, Rossetti ML. Tuberculosis in a southern Brazilian prison. Mem Inst Oswaldo Cruz 2012; 107(7):909-915.

19. Nogueira PA, Abrahão RMCM, Galesi VNM. Tuberculosis and latenttuberculosis in prison inmates. Rev Saude Publica 2012; 46(1):119-127.

20. Nogueira PA, Abrahão RMCM, Galesi VNM. Infecção tuberculosa latente em profissionais contatos e não contatos de detentos de duas penitenciárias do Estado de São Paulo, Brasil, 2008. Rev Bras Epidemiol 2011; 14(3):486-494.

21. Vieira AA, Ribeiro SA, Siqueira AM, Galesi VNM, Santos LAR, Golub JE. Prevalence of patients with respiratory symptoms through active case finding and diagnosis of pulmonary tuberculosis among prisoners and related predictors in a jail in the city of Carapicuíba, Brazil. Rev Bras Epidemiol 2010; 13(4):641-650.

22. Zanini RJ, Valença MS, Carrion LL, Silva LV, von Groll A, Silva PA. Respiratory symptoms and active tuberculosis in a prison in Southern Brazil: Associated epidemiologic variables. Rev Epidemiol Control Infect 2013; 3(4):128-133.

23. Pereira CC, Borges TS, Daronco A, Valim ARM, Carneiro M, Becker D, Possuelo LG. Prevalência e sintomáticos respiratórios e tuberculose ativa em uma Penitenciária do Sul do Brasil. Rev Epidemiol Control Infect 2013; 3(3):99-104.

24. Macedo LR, Reis SB, Riley LW, Maciel EL. Treatment outcomes of tuberculosis patients in Brazilian prisons: a polytomous regression analysis. Int J Tuberc Lung Dis 2013; 17(11):1427-34

25. Ferreira Júnior S, Oliveira HB, Marin-Léon L. Conhecimento, atitudes e práticas sobre tuberculose em prisões e no serviço público de saúde. Rev Bras Epidemiol 2013; 16(1):100-113.

26. Souza KMJ, Villa TCS, Assolini FEP, Beraldo AA, França UM, Protti ST, Palha PF.Atraso no diagnóstico da tuberculose em sistema prisional: a experiência do doente apenado. Text Context Nursing2012; 21(1):17-25.

27. Pedro HSP, Nardi SMT, Pereira MIF, Goloni MRA, Pires FC, Tolentino FM, Oliveira RS, Rossit ARB. Mycobacterium tuberculosis detection in the penitentiary system. Rev. Patol. Trop. 2011; 40(4):287-95.

28. Moreira-Oliveira HB, Oliveira PF, Stehling EG, Rocha MMM, Aily DCG, Brocchi1 M, Silveira WD. Molecular Genotyping and Epidemiology of Mycobacterium tuberculosis Isolates Obtained from Inmates of Correctional Institutions of Campinas, Southeast Brazil. Braz J Infect Dis 2008; 12(6):487-93.

29. Legrand J, Sanchez A, Le Pont F, Camacho L, Larouze B. Modeling the Impact of Tuberculosis Control Strategies in Highly Endemic Overcrowded Prisons. PLoS ONE 2008; 3(5):e2100. 
30. Fournet N, Sanchez A, Massari V, Penna L, Natal S, Biondi E, Larouzé B. Development and evaluation of tuberculosis screening scores in Brazilian prisons. Public Health 2006; 120(10):976-983.

31. Oliveira HB, Cardoso JC. Tuberculose no sistema prisional de Campinas, São Paulo, Brasil. Rev Panam Salud Publica 2004; 15(3):194-199.

32. World Health Organization (WHO). Tuberculosis control in prisons. A manual for programme managers. Geneva: WHO; 2000.

33. Zenner D, Southern J, van Hest R, de Vries,Stagg HR, Antoine D, Abubakar I. Active case finding for tuberculosis among high-risk groups in low-incidence countries. Int J Tuberc Lung Dis 2013; 17(5):573-582.

34. Golub JE, Mohan CI, Comstock GW, Chaisson RE. Active case finding of tuberculosis: historical perspective and future prospects. Int J Tuberc Lung Dis 2005; 9(11):1183-1203.

35. Sudre P, ten Dam G, Kochi A. Tuberculosis: a global overview of the situation today. Bull World Health Organ 1992; 70(2):149-159.

36. Salive ME, Vlahov D, Brewer TF. Coinfection with tuberculosis and HIV-1 in male prison inmates. Public Health Rep 1990; 105(3):307-310.

37. Anderson KM, Keith EP, Norsted SW. Tuberculosis screening in Washington State male correctional facilities. Chest 1986; 89(6):817-821.

38. Severo KGP, Oliveira JS, Carneiro M, Valim ARM, Krummenauer EC, Possuelo LG. Latent tuberculosis in nursing professionals of a Brazilian hospital. J Occup Med Toxicol 2011; 6(1):15.

39. Braga JU, Barreto AM, Hijjar MA. Inquérito epidemiológico da resistência às drogas usadas no tratamento da tuberculose no Brasil 1995-97, IERDTB. Parte III: principais resultados. Bol Pneumol Sanit 2003; 11(1):76-81.
40. Brasil. Ministério da Saúde (MS). Secretaria de Vigilância em Saúde. II Inquérito Nacional de Resistência a Drogas em Tuberculose: protocolo. Brasília: MS; 2005.

41. World Health Organization (WHO). Multidrug and Extensively drug-resistence TB (M/XDR TB). 2010 Global Report on Surveillance and Response. Geneva: WHO; 2010.

42. Kritski AL. Multidrug-resistant tuberculosis emergence: a renewed challenge. J Bras Pneumol 2010; 36(2):157-158.

43. World Health Organization (WHO). Global Tuberculosis Report 2000. Geneva: WHO; 2000.

44. World Health Organization (WHO). Global Tuberculosis Control 2011. Geneva: WHO; 2011.

45. Supply P, Mazars E, Lesjean S, Vincent V, Gicquel B, Locht C. Variable human minisatellite-like regions in the Mycobacterium tuberculosis genome. Mol. Microbiol. 2000; 36(3):762-771.

46. Burgos MV, Pym AS. Molecular epidemiology of tuberculosis. Eur Respir J 2002; 20:54s-65s.

Artigo apresentado em 18/03/2015

Aprovado em 17/09/2015

Versão final apresentada em 19/09/2015 